\title{
XH-stretching overtone transitions calculated using explicitly correlated coupled cluster methods
}

\author{
Joseph R. Lane ${ }^{1}$ and Henrik G. Kjaergaard ${ }^{2, a)}$ \\ ${ }^{1}$ Department of Chemistry, University of Waikato, Private Bag 3105, Hamilton 3240, New Zealand \\ ${ }^{2}$ Department of Chemistry, University of Copenhagen, Universitetsparken 5, DK-2100, \\ Copenhagen $\varnothing$, Denmark
}

(Received 26 January 2010; accepted 3 April 2010; published online 6 May 2010)

\begin{abstract}
We have calculated $\mathrm{XH}$-stretching (where $\mathrm{X}=\mathrm{O}, \mathrm{C}, \mathrm{F}, \mathrm{Cl}$ ) fundamental and overtone transitions for three diatomics and a few small molecules using a local mode model. The potential energy curves and dipole moment functions are calculated using the recently developed explicitly correlated coupled cluster with single doubles and perturbative triples theory [CCSD(T)-F12] with the associated VXZ-F12 (where X=D, T, Q) basis sets. We find that the basis set convergence of calculated frequencies and oscillator strengths obtained with the explicitly correlated method is much more rapid than with conventional $\operatorname{CCSD}(\mathrm{T})$ and the Dunning type correlation consistent basis sets. Furthermore, $\operatorname{CCSD}(\mathrm{T})-\mathrm{F} 12$ frequencies and oscillator strengths obtained with the VTZ-F12 and VQZ-F12 basis sets are found to be in excellent agreement with the CCSD(T) complete basis set limit. We find that comparison of $\operatorname{CCSD}(\mathrm{T})-\mathrm{F} 12$ frequencies with experiment is less good. The inclusion of explicit correlation exposes the inherent error of the $\operatorname{CCSD}(\mathrm{T})$ method to overestimate vibrational frequencies, which is normally compensated by basis set incompleteness error. As a consequence, we suggest that conventional $\operatorname{CCSD}(\mathrm{T})$ in combination with the aug-cc-pVTZ or aug-cc-pVQZ basis sets is likely to yield calculated XH-stretching frequencies in closest agreement with experiment. (C) 2010 American Institute of Physics. [doi:10.1063/1.3408192]
\end{abstract}

\section{INTRODUCTION}

Coupled cluster theory including singles doubles and perturbative triples excitations $[\operatorname{CCSD}(\mathrm{T})]$ has emerged as the so-called "gold standard" method of modern computational chemistry that can be routinely applied to small systems of chemical interest. ${ }^{1-3}$ The computational demands of the $\operatorname{CCSD}(\mathrm{T})$ method are very high (scaling nominally $n^{7}$, where $n$ is the size of the system) and because extensive basis sets are necessary to reliably achieve accurate results, it is impracticable for larger systems. However, the painfully slow convergence of electron correlation energy with increasing basis set size can be significantly improved by including a small number of terms to the wave function that depend explicitly on the interelectronic distance $r_{12}{ }^{4}$ The recently developed explicitly correlated CCSD(T)-F12 methods have shown to give results using a triple- $\zeta$ basis set that are better than conventional $\operatorname{CCSD}(\mathrm{T})$ with a quintuple- $\zeta$ basis set. ${ }^{5-7}$ Very recently, fundamental frequencies calculated using a vibrational configuration interaction (VCI) anharmonic vibrational model with $\mathrm{CCSD}(\mathrm{T})-\mathrm{F} 12$ force constants were found to have an absolute mean deviation of just $4 \mathrm{~cm}^{-1}$ when compared to experiment. ${ }^{8}$

Overtone vibrational spectra are very sensitive to bond properties and can be used to study subtle effects in molecular structure and conformation. ${ }^{9}$ As higher regions of the potential energy curve are probed, differences that might appear small in the fundamental region become much more apparent for overtone transitions. Vibrational spectra in the near-

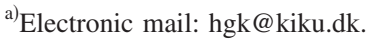

infrared and visible regions are dominated by $\mathrm{XH}$-stretching overtone transitions, where $\mathrm{X}$ is a heavy atom such as $\mathrm{C}, \mathrm{O}$, and N. ${ }^{9-11}$ The vibrations of XH-stretching oscillators are highly anharmonic and can be described both theoretically and experimentally using an anharmonic oscillator local mode model. ${ }^{9}$ Earlier theoretical predictions of $\mathrm{XH}-$ stretching transitions used input from low level $a b$ initio and density functional calculations that were empirically scaled to improve agreement with experiment. ${ }^{12}$ In more recent years, XH-stretching transitions of several small molecules including ethylene glycol, peroxynitrous acid, hydroxymethyl hydroperoxide, sulfuric acid, methanesulfonic acid, ethanol, ethanethiol, tert-butanol, and tert-butylthiol have been successfully calculated entirely $a b$ initio with potential energy and dipole moment curves obtained with the $\operatorname{CCSD}(\mathrm{T})$ method. ${ }^{13-20}$ For many of these systems, a triple- $\zeta$ correlation consistent basis set was pragmatically used as larger basis sets were not considered practicable at the $\operatorname{CCSD}(\mathrm{T})$ level of theory.

In this work, we investigate the basis set convergence of $\mathrm{XH}$-stretching fundamental and overtone transitions of hydroxyl radical $(\mathrm{OH})$, hydrogen fluoride $(\mathrm{HF})$, hydrogen chloride $(\mathrm{HCl})$, formic acid $(\mathrm{HCOOH})$, methanol $\left(\mathrm{CH}_{3} \mathrm{OH}\right)$, nitric acid $\left(\mathrm{HNO}_{3}\right)$, and water dimer $\left(\mathrm{H}_{2} \mathrm{O}-\mathrm{H}_{2} \mathrm{O}\right)$ calculated with a one-dimensional (1D) local mode model at the $\operatorname{CCSD}(\mathrm{T})$ level of theory. The potential energy and dipole moment curves are calculated using both conventional $\operatorname{CCSD}(\mathrm{T})$ theory and explicitly correlated $\operatorname{CCSD}(\mathrm{T})-\mathrm{F} 12$ theory with appropriate correlation consistent basis sets. For the three diatomic systems, we compare the basis set conver- 
gence of the calculated $\mathrm{XH}$-stretching frequencies and oscillator strengths to results obtained at the complete basis set (CBS) limit and to experiment. For the polyatomic systems, we consider the relative basis set convergence of the conventional and explicitly correlated $\mathrm{XH}$-stretching transitions and compare both sets of calculated results to experiment.

\section{THEORETICAL METHODS}

We have optimized the geometries of $\mathrm{OH}, \mathrm{HF}, \mathrm{HCl}$, $\mathrm{HCOOH}, \mathrm{CH}_{3} \mathrm{OH}, \mathrm{HNO}_{3}$, and $\mathrm{H}_{2} \mathrm{O}-\mathrm{H}_{2} \mathrm{O}$ with conventional $\operatorname{CCSD}(\mathrm{T})$ ab initio theory using Dunning type correlation consistent basis sets. With a suitably large basis set, the $\operatorname{CCSD}(\mathrm{T})$ method has been shown to give accurate geometries of small systems that are in excellent agreement with experiment. ${ }^{1-3}$ We have used the aug-cc-pVXZ basis sets (where $X=D, T, Q, 5,6)$ for first row elements and the aug-cc-pV $(X+d) Z$ basis sets (where $X=D, T, Q, 5,6)$ for all second row elements. ${ }^{21,22}$ For brevity, we refer to these basis sets as AVXZ (where X=D, T, Q , 5,6) whereby it is assumed that second row elements have the additional tight $\mathrm{d}$ basis functions. For $\mathrm{H}_{2} \mathrm{O}-\mathrm{H}_{2} \mathrm{O}$, we have also constructed basis sets where only the oxygen atoms are augmented with diffuse basis functions and refer to these as $\mathrm{A}^{\prime} \mathrm{VXZ}$ (where $\mathrm{X}$ $=\mathrm{D}, \mathrm{T}, \mathrm{Q}, 5){ }^{23}$

We have also optimized the geometries of $\mathrm{OH}, \mathrm{HF}, \mathrm{HCl}$, $\mathrm{HCOOH}, \mathrm{CH}_{3} \mathrm{OH}, \mathrm{HNO}_{3}$, and $\mathrm{H}_{2} \mathrm{O}-\mathrm{H}_{2} \mathrm{O}$ with the recently developed explicitly correlated $\operatorname{CCSD}(\mathrm{T})-\mathrm{F} 12$ methods as implemented in MOLPRO 2008.1. ${ }^{7,24}$ We have used the VXZ$\mathrm{F} 12$ orbital basis sets (where $\mathrm{X}=\mathrm{D}, \mathrm{T}, \mathrm{Q}$ ) of Peterson et al. that have been specifically optimized for use with explicitly correlated F12 methods. ${ }^{25}$ For a given cardinal number, the VXZ-F12 basis sets have been designed to be of similar size to the equivalent aug-cc-pVXZ and aug-cc-pV $(\mathrm{X}+\mathrm{d}) \mathrm{Z}$ basis sets. Density fitting approximations ${ }^{26,27}$ were used in all explicitly correlated calculations using the VXZ/JKFIT (where $\mathrm{X}=\mathrm{D}, \mathrm{T}, \mathrm{Q}$ ) and the AVXZ/MP2FIT (where $\mathrm{X}=\mathrm{D}, \mathrm{T}, \mathrm{Q}$ ) auxiliary basis sets of Weigend et al. ${ }^{28,29}$ We have used the resolution of the identity (RI) auxiliary basis sets of Yousaf et al. for all RI approximations. ${ }^{30}$ The default CCSD-F12 correlation factor $\left[(1 / \beta) e^{-\beta r_{12}}\right.$, where $\left.\beta=1\right]$ was used in all explicitly correlated calculations.

Two different approximations are available for solving the CCSD(T)-F12 energies in MOLPRO 2008.1, denoted $\operatorname{CCSD}(\mathrm{T})-\mathrm{F} 12 \mathrm{a}$ and $\operatorname{CCSD}(\mathrm{T})-\mathrm{F} 12 \mathrm{~b}$. Overall, we find that results obtained with the two approximations are in very good agreement with each other, with the $\operatorname{CCSD}(\mathrm{T})-\mathrm{F} 12 \mathrm{~b}$ frequencies consistently slightly higher than the $\operatorname{CCSD}(\mathrm{T})$ F12a frequencies. We limit discussion in the manuscript to $\operatorname{CCSD}(\mathrm{T})$-F12b results, which appear to converge more smoothly to the CBS limit, in agreement with previous work. ${ }^{31}$ The equivalent $\operatorname{CCSD}(\mathrm{T})$-F12a results are included in the supplementary deposit. ${ }^{32}$

The dimensionless oscillator strength $f$ of a transition from the vibrational ground state $|0\rangle$ to a vibrationally excited state $|v\rangle$ is given by ${ }^{33,34}$

$$
f_{v 0}=4.702 \times 10^{-7}\left[\mathrm{~cm} \mathrm{D}^{-2}\right] \widetilde{\nu}_{v 0}\left|\vec{\mu}_{v 0}\right|^{2},
$$

where $\widetilde{\nu}_{v 0}$ is the vibrational wavenumber of the transition in $\mathrm{cm}^{-1}$ and $\vec{\mu}_{v 0}=\langle v|\vec{\mu}| 0\rangle$ is the transition dipole moment matrix element in Debye (D).

We calculate the $\mathrm{XH}$-stretching frequencies and oscillator strengths for $\mathrm{OH}, \mathrm{HF}, \mathrm{HCl}, \mathrm{HCOOH}, \mathrm{CH}_{3} \mathrm{OH}, \mathrm{HNO}_{3}$, and $\mathrm{H}_{2} \mathrm{O}-\mathrm{H}_{2} \mathrm{O}$ with a 1D local mode vibrational model. ${ }^{9,10,14}$ The 1D Schrödinger equation is solved numerically using a finite element method to give both the vibrational energy levels and wave functions. ${ }^{35}$ The potential energy curve used for this covers the range from -0.3 to $0.8 \AA$ in $0.025 \AA$ steps around equilibrium. This ensures converged energy levels, better than $0.1 \mathrm{~cm}^{-1}$, and wave functions ( $f$ better than $0.1 \%$ ) for $v \leq 5$ when tested for range and step size in the potential energy curve.

The transition dipole matrix element of Eq. (1) can be expanded as

$\langle v|\vec{\mu}| 0\rangle=\frac{\partial \vec{\mu}}{\partial q}\langle v|q| 0\rangle+\frac{1}{2} \frac{\partial^{2} \vec{\mu}}{\partial q^{2}}\left\langle v\left|q^{2}\right| 0\right\rangle+\frac{1}{6} \frac{\partial^{3} \vec{\mu}}{\partial q^{3}}\left\langle v\left|q^{3}\right| 0\right\rangle+\ldots$,

where $q$ is the internal vibrational displacement coordinate. The integrals $\left\langle v\left|q^{n}\right| 0\right\rangle$ required for the transition dipole moment are evaluated by trapezoidal numeric integration. The dipole moment coefficients are found from a sixth order polynomial fit to a 15 point dipole moment curve calculated over values of $q$ from -0.3 to $0.4 \AA$ in $0.05 \AA$ steps. ${ }^{36,37}$ These dipole moment coefficients provide oscillator strengths that are converged to better than $1 \%$ when compared to larger dipole moment curves. ${ }^{13}$

For the diatomics, we have also constructed conventional CCSD(T)/CBS limit potential energy curves. We have extrapolated the $\operatorname{CCSD}(\mathrm{T})$ energies at each point with the following two parameter extrapolation for the correlation energy:

$$
E_{X Y}^{\text {corr }}=\frac{X^{3} E_{X}^{\text {corr }}-Y^{3} E_{Y}^{\text {corr }}}{X^{3}-Y^{3}},
$$

where $X$ and $Y$ are the cardinal numbers of the two basis sets and $E_{X}^{\text {corr }}$ and $E_{Y}^{\text {corr }}$ are the corresponding correlation energies. ${ }^{38,39}$ The extrapolated correlation energy, $E_{56}^{\text {corr }}$ is added to the HF/AV6Z energy to give an estimate of the $\operatorname{CCSD}(\mathrm{T}) / \mathrm{CBS}$ energy. All energies are obtained at displacements from the CCSD(T)/AV6Z optimized bond length. The $\operatorname{CCSD}(\mathrm{T})$ and $\operatorname{CCSD}(\mathrm{T})$-F12 dipole moment at each geometry is calculated using a finite field approach with a field strength of \pm 0.0005 a.u. We construct CCSD(T)/CBS dipole moment curves by extrapolating the correlation energy using Eq. (3) under the influence of both positive and negative electric fields and then evaluate the dipole moment. ${ }^{40}$

To investigate the appropriateness of our 1D local mode vibrational mode for the polyatmomic systems, we have also calculated the fundamental and overtone vibrational frequencies of $\mathrm{HCOOH}, \mathrm{CH}_{3} \mathrm{OH}, \mathrm{HNO}_{3}$, and $\mathrm{H}_{2} \mathrm{O}-\mathrm{H}_{2} \mathrm{O}$ using second order vibrational perturbation theory (VPT2). The VPT2 vibrational model has been show to give accurate fundamental, overtone, and combination vibrational frequencies for 
TABLE I. Conventional CCSD(T) and explicitly correlated CCSD(T)-F12b calculated and experimental frequencies (in $\mathrm{cm}^{-1}$ ) for hydroxyl radical.

\begin{tabular}{llllll}
\hline \hline Basis set & $\Delta v=1$ & $\Delta v=2$ & $\Delta v=3$ & $\Delta v=4$ & $\Delta v=5$ \\
\hline AVDZ & 3510 & 6849 & 10022 & 13030 & 15871 \\
AVTZ & 3554 & 6947 & 10177 & 13245 & 16151 \\
AVQZ & 3572 & 6979 & 10223 & 13306 & 16227 \\
AV5Z & 3575 & 6985 & 10233 & 13320 & 16245 \\
AV6Z & 3575 & 6986 & 10235 & 13322 & 16249 \\
CBS $^{\text {a }}$ & 3576 & 6987 & 10236 & 13325 & 16253 \\
VDZ-F12 & 3576 & 6987 & 10235 & 13321 & 16244 \\
VTZ-F12 & 3574 & 6983 & 10230 & 13315 & 16240 \\
VQZ-F12 & 3576 & 6989 & 10239 & 13328 & 16256 \\
Expt. $^{\text {b }}$ & 3569.61 & 6973.68 & 10214.05 & 13291.82 & 16207.12 \\
\hline \hline
\end{tabular}

${ }^{\mathrm{a}}$ Extrapolation to the CBS limit utilizes AV5Z and AV6Z results.

${ }^{\mathrm{b}}$ Experimental frequencies from HITRAN (Ref. 62).

several small molecules at the $\operatorname{CCSD}(\mathrm{T})$ level of theory with ANO and correlation consistent basis sets. ${ }^{41,42}$ Anharmonic VPT2 frequencies were performed using $\mathrm{CFOUR}^{43}$ with the following convergence threshold criteria: $\mathrm{SCF}$ CONV=1 $\times 10^{-9}$ a.u., CC_CONV $=1 \times 10^{-10}$ a.u., LINEQ_CONV $=1 \times 10^{-10}$ a.u., and GEO_CONV $=1 \times 10^{-10}$ a.u.

All coupled cluster calculations assume a frozen core (C:1s; N:1s; O:1s;, S:1s,2s,2p; Cl:1s,2s,2p) and unless otherwise specified were performed using MOLPRO 2008.1. ${ }^{24}$ The optimization threshold criteria in MOLPRO was set to gradient $=1 \times 10^{-6}$ a.u., stepsize $=1 \times 10^{-6}$ a.u., energy $=1$ $\times 10^{-8}$ a.u. The single point threshold criterion was set to: energy $=1 \times 10^{-9}$ a.u., orbital $=1 \times 10^{-8}$ a.u., coefficient $=1$ $\times 10^{-8}$ a.u.

\section{RESULTS AND DISCUSSION}

\section{A. Diatomics}

In Tables I-III we present fundamental and overtone vibrational frequencies for $\mathrm{OH}, \mathrm{HF}$, and $\mathrm{HCl}$ obtained with both conventional and explicitly correlated $\operatorname{CCSD}(\mathrm{T})$ potential energy curves. If we first consider the conventional $\operatorname{CCSD}(\mathrm{T})$ results, we find that in general the vibrational frequencies of the three diatomics converge upwards to the $\operatorname{CCSD}(\mathrm{T}) / \mathrm{CBS}$ limit as we increase the cardinal number of the basis set from AVDZ to AV6Z. This result is consistent with previous basis set investigations of the harmonic frequencies of diatomic molecules obtained with the $\operatorname{CCSD}(\mathrm{T})$ method. ${ }^{44,45}$ For $\mathrm{OH}$ and HF, convergence of the fundamental and overtone frequencies is slow but proceeds monotonically from AVDZ to AV6Z. For $\mathrm{HCl}$, the calculated frequencies appear to converge a little more rapidly although there is some small oscillation between the AVQZ-AV6Z results. This oscillation in the $\mathrm{HCl}$ vibrational frequencies is due to a small oscillation in the optimized $\mathrm{CCSD}(\mathrm{T}) \mathrm{HCl}$ bond length with the AVQZ-AV6Z basis sets.

Not surprisingly, the absolute error between frequencies obtained with a given basis set and the CBS limit is smallest for the fundamental transitions and becomes increasingly large for the higher overtones. For example, the AVDZ OH fundamental frequency is underestimated by $66 \mathrm{~cm}^{-1}$, whereas the AVDZ OH fourth overtone is underestimated by $382 \mathrm{~cm}^{-1}$. However, in relative terms, the percentage error also generally increases slightly as the quanta of excitation increases. For example, the AVDZ OH fundamental frequency is underestimated by $1.9 \%$ and the AVDZ OH fourth overtone is underestimated by $2.5 \%$. This suggests that the higher parts of the potential energy curve have greater basis set requirements than points closer to equilibrium.

If we now consider the explicitly correlated results, we find that the calculated fundamental and overtone vibrational frequencies oscillate as the cardinal number of the basis set

TABLE II. Conventional CCSD(T) and explicitly correlated CCSD(T)-F12b calculated and experimental frequencies $\left(\mathrm{in} \mathrm{cm}^{-1}\right.$ ) for hydrogen fluoride.

\begin{tabular}{llllll}
\hline \hline Basis set & $\Delta v=1$ & $\Delta v=2$ & $\Delta v=3$ & $\Delta v=4$ & $\Delta v=5$ \\
\hline AVDZ & 3891 & 7604 & 11148 & 14529 & 17752 \\
AVTZ & 3952 & 7734 & 11349 & 14800 & 18091 \\
AVQZ & 3965 & 7759 & 11384 & 14847 & 18150 \\
AV5Z & 3966 & 7760 & 11387 & 14851 & 18156 \\
AV6Z & 3966 & 7760 & 11387 & 14852 & 18157 \\
CBS $^{\mathrm{a}}$ & 3966 & 7759 & 11387 & 14851 & 18157 \\
VDZ-F12 $_{\text {VTZ-F12 }}^{3971}$ & 7771 & 11405 & 14877 & 18190 \\
VQZ-F12 $_{\text {Expt. }}^{\text {b }}$ & 3964 & 7757 & 11384 & 14847 & 18151 \\
\hline \hline
\end{tabular}

${ }^{\mathrm{a}}$ Extrapolation to the CBS limit utilizes AV5Z and AV6Z results.

${ }^{\mathrm{b}}$ Experimental frequencies from HITRAN (Ref. 62). 
TABLE III. Conventional CCSD(T) and explicitly correlated CCSD(T)-F12b calculated and experimental frequencies $\left(\right.$ in $\mathrm{cm}^{-1}$ ) for hydrogen chloride.

\begin{tabular}{|c|c|c|c|c|c|}
\hline Basis set & $\Delta v=1$ & $\Delta v=2$ & $\Delta v=3$ & $\Delta v=4$ & $\Delta v=5$ \\
\hline AVDZ & 2879 & 5651 & 8317 & 10877 & 13330 \\
\hline AVTZ & 2890 & 5674 & 8353 & 10927 & 13398 \\
\hline AVQZ & 2891 & 5678 & 8362 & 10943 & 13422 \\
\hline AV5Z & 2892 & 5680 & 8365 & 10948 & 13428 \\
\hline AV6Z & 2891 & 5679 & 8364 & 10947 & 13428 \\
\hline $\mathrm{CBS}^{\mathrm{a}}$ & 2890 & 5676 & 8361 & 10944 & 13425 \\
\hline VDZ-F12 & 2906 & 5708 & 8407 & 11003 & 13496 \\
\hline VTZ-F12 & 2891 & 5679 & 8364 & 10947 & 13428 \\
\hline VQZ-F12 & 2892 & 5680 & 8366 & 10949 & 13431 \\
\hline Expt. $^{b}$ & 2885.9 & 5668.0 & 8346.9 & 10923.1 & 13396.5 \\
\hline
\end{tabular}

${ }^{\mathrm{a}}$ Extrapolation to the CBS limit utilizes AV5Z and AV6Z results.

${ }^{\mathrm{b}}$ Experimental frequencies from HITRAN (Ref. 62).

increases from VDZ-F12 to VQZ-F12. For all three diatomics, the VDZ-F12 vibrational frequencies are higher than the VTZ-F12 vibrational frequencies, which in turn are marginally lower than the VQZ-F12 vibrational frequencies. However, it should be noted that the oscillation from VDZ-F12 to VQZ-F12 is significantly smaller than the monotonic variation in the vibrational frequencies from AVDZ to AVQZ observed with conventional CCSD(T). Furthermore, fundamental and overtone frequencies obtained with a given cardinal number and the explicitly correlated CCSD(T)-F12b method are in much better agreement with the CCSD(T)/CBS limit than results obtained with conventional $\operatorname{CCSD}(\mathrm{T})$. Impressively for all three diatomics, CCSD(T)-F12b vibrational frequencies obtained with the VTZ-F12 basis set are of comparable accuracy [with respect to the $\operatorname{CCSD}(\mathrm{T}) / \mathrm{CBS}$ limit] to $\operatorname{CCSD}(\mathrm{T}) / \mathrm{AV} 5 \mathrm{Z}$ results and are much less computationally demanding.

Now that we have established the basis set convergence of both the conventional and explicitly correlated $\operatorname{CCSD}(\mathrm{T})$ vibrational frequencies, it is pertinent to compare our calculated results to experiment. We find that the CCSD(T)/CBS fundamental frequencies are overestimated by $4-6 \mathrm{~cm}^{-1}$ $(0.11 \%-0.18 \%)$ with respect to experiment. This result is comparable to the $3-14 \mathrm{~cm}^{-1}$ overestimate of $\operatorname{CCSD}(\mathrm{T})$ harmonic frequencies with respect to experiment that was previously observed for the diatomics $\mathrm{HF}, \mathrm{N}_{2}, \mathrm{~F}_{2}$, and $\mathrm{CO} .{ }^{45}$
The absolute and relative error of the CCSD(T)/CBS frequencies increases as the quanta of excitation increases. For the fourth overtone, the $\operatorname{CCSD}(\mathrm{T}) / \mathrm{CBS}$ frequencies are overestimated by $26-46 \mathrm{~cm}^{-1}(0.14 \%-0.28 \%)$

For $\mathrm{OH}$ and $\mathrm{HF}$, the conventional $\mathrm{CCSD}(\mathrm{T})$ vibrational frequencies obtained with the AVDZ and AVTZ basis sets are underestimated with respect to experiment while the AVQZAV6Z results are overestimated. For $\mathrm{HCl}$, only the AVDZ vibrational frequencies are underestimated with the AVTZAV6Z results overestimated. As a consequence of the rapid basis set convergence of the explicitly correlated $\operatorname{CCSD}(\mathrm{T})$ F12b method, even XH-stretching frequencies obtained with the VDZ-F12 basis set are overestimated when compared to the experimental values for $\mathrm{OH}, \mathrm{HF}$, and $\mathrm{HCl}$. The inclusion of explicit correlation appears to expose the inherent error of the $\operatorname{CCSD}(\mathrm{T})$ method for calculating vibrational frequencies that is normally compensated to a certain extent by basis set incompleteness error. Fortuitously, we find conventional $\operatorname{CCSD}(\mathrm{T}) / \mathrm{AVQZ}$ frequencies for $\mathrm{OH}$ and $\mathrm{HF}$ and conventional $\operatorname{CCSD}(\mathrm{T}) / \mathrm{AVTZ}$ frequencies of $\mathrm{HCl}$ are in closest agreement with experiment.

In Tables IV-VI we present fundamental and overtone vibrational oscillator strengths for $\mathrm{OH}, \mathrm{HF}$, and $\mathrm{HCl}$ obtained with both conventional and explicitly correlated $\operatorname{CCSD}(\mathrm{T})$ potential energy and dipole moment curves. In comparison with the vibrational frequencies, the convergence of calcu-

TABLE IV. Conventional CCSD(T) and explicitly correlated CCSD(T)-F12b calculated and experimental oscillator strengths for hydroxyl radical.

\begin{tabular}{lccccc}
\hline \hline Basis set & $\Delta v=1$ & $\Delta v=2$ & $\Delta v=3$ & $\Delta v=4$ & $\Delta v=5$ \\
\hline AVDZ & $9.3 \times 10^{-7}$ & $2.2 \times 10^{-7}$ & $1.1 \times 10^{-8}$ & $8.4 \times 10^{-10}$ & $7.9 \times 10^{-11}$ \\
AVTZ & $1.4 \times 10^{-6}$ & $2.4 \times 10^{-7}$ & $1.1 \times 10^{-8}$ & $6.4 \times 10^{-10}$ & $5.4 \times 10^{-11}$ \\
AVQZ & $1.6 \times 10^{-6}$ & $2.5 \times 10^{-7}$ & $1.1 \times 10^{-8}$ & $6.4 \times 10^{-10}$ & $5.5 \times 10^{-11}$ \\
AV5Z & $1.7 \times 10^{-6}$ & $2.5 \times 10^{-7}$ & $1.0 \times 10^{-8}$ & $6.3 \times 10^{-10}$ & $5.4 \times 10^{-11}$ \\
AV6Z & $1.7 \times 10^{-6}$ & $2.5 \times 10^{-7}$ & $1.0 \times 10^{-8}$ & $6.2 \times 10^{-10}$ & $5.3 \times 10^{-11}$ \\
CBS $^{\text {a }}$ & $1.7 \times 10^{-6}$ & $2.5 \times 10^{-7}$ & $1.0 \times 10^{-8}$ & $6.3 \times 10^{-10}$ & $5.3 \times 10^{-11}$ \\
VDZ-F12 & $1.7 \times 10^{-6}$ & $2.7 \times 10^{-7}$ & $1.1 \times 10^{-8}$ & $6.9 \times 10^{-10}$ & $6.1 \times 10^{-11}$ \\
VTZ-F12 & $1.8 \times 10^{-6}$ & $2.6 \times 10^{-7}$ & $1.1 \times 10^{-8}$ & $6.2 \times 10^{-10}$ & $5.2 \times 10^{-11}$ \\
VQZ-F12 & $1.8 \times 10^{-6}$ & $2.5 \times 10^{-7}$ & $1.0 \times 10^{-8}$ & $6.2 \times 10^{-10}$ & $5.3 \times 10^{-11}$ \\
Expt. $^{\text {b }}$ & $1.17 \times 10^{-6}$ & $2.07 \times 10^{-7}$ & $1.07 \times 10^{-8}$ & $7.54 \times 10^{-10}$ & $7.26 \times 10^{-11}$ \\
\hline \hline
\end{tabular}

${ }^{\mathrm{a}}$ Extrapolation to the CBS limit utilizes AV5Z and AV6Z results.

${ }^{\mathrm{b}}$ Experimental oscillator strengths from HITRAN (Ref. 62). 
TABLE V. Conventional CCSD(T) and explicitly correlated CCSD(T)-F12b calculated and experimental oscillator strengths for hydrogen fluoride.

\begin{tabular}{lccccc}
\hline \hline Basis set & $\Delta v=1$ & $\Delta v=2$ & $\Delta v=3$ & $\Delta v=4$ & $\Delta v=5$ \\
\hline AVDZ & $1.7 \times 10^{-5}$ & $5.7 \times 10^{-7}$ & $1.9 \times 10^{-8}$ & $1.1 \times 10^{-10}$ & $9.0 \times 10^{-11}$ \\
AVTZ & $1.8 \times 10^{-5}$ & $5.7 \times 10^{-7}$ & $1.6 \times 10^{-8}$ & $7.2 \times 10^{-10}$ & $4.6 \times 10^{-11}$ \\
AVQZ & $1.9 \times 10^{-5}$ & $5.7 \times 10^{-7}$ & $1.6 \times 10^{-8}$ & $8.2 \times 10^{-10}$ & $6.0 \times 10^{-11}$ \\
AV5Z & $1.9 \times 10^{-5}$ & $5.7 \times 10^{-7}$ & $1.6 \times 10^{-8}$ & $8.3 \times 10^{-10}$ & $6.1 \times 10^{-11}$ \\
AV6Z & $1.9 \times 10^{-5}$ & $5.7 \times 10^{-7}$ & $1.6 \times 10^{-8}$ & $8.3 \times 10^{-10}$ & $6.1 \times 10^{-11}$ \\
CBS $^{\text {a }}$ & $1.9 \times 10^{-5}$ & $5.7 \times 10^{-7}$ & $1.6 \times 10^{-8}$ & $8.2 \times 10^{-10}$ & $5.9 \times 10^{-11}$ \\
VDZ-F12 $_{\text {VTZ-F12 }}$ & $1.9 \times 10^{-5}$ & $5.9 \times 10^{-7}$ & $1.7 \times 10^{-8}$ & $9.1 \times 10^{-10}$ & $7.3 \times 10^{-11}$ \\
VQZ-F12 & $1.9 \times 10^{-5}$ & $5.8 \times 10^{-7}$ & $1.6 \times 10^{-8}$ & $8.2 \times 10^{-10}$ & $5.9 \times 10^{-11}$ \\
Expt. $^{\text {b }}$ & $1.9 \times 10^{-5}$ & $5.8 \times 10^{-7}$ & $1.6 \times 10^{-8}$ & $8.4 \times 10^{-10}$ & $6.2 \times 10^{-11}$ \\
\hline \hline
\end{tabular}

${ }^{\mathrm{a}}$ Extrapolation to the CBS limit utilizes AV5Z and AV6Z results.

${ }^{\mathrm{b}}$ Experimental oscillator strengths from HITRAN (Ref. 62).

lated oscillator strengths is not consistent across the quanta of excitation. For example, the fundamental oscillator strengths generally converge upwards whereas the oscillator strengths of the higher overtones generally converge downwards with increasing basis set size. However, the calculated oscillator strengths do appear to converge more rapidly than the calculated frequencies as the cardinal number of the basis set is increased, which suggests that the dipole moment curve is less sensitive to basis set size than the potential energy curve. With conventional $\operatorname{CCSD}(\mathrm{T})$, oscillator strengths obtained with the AVQZ basis set are essentially converged to the CCSD(T)/CBS limit. The basis set convergence of the explicitly correlated $\operatorname{CCSD}(\mathrm{T})-\mathrm{F} 12 \mathrm{~b}$ method is a little better, with oscillator strengths obtained using the VTZF12 basis found to be in excellent agreement with the CCSD(T)/CBS limit. Notably, the fundamental, first and second overtone oscillator strengths of the three diatomics are also essentially converged to the $\operatorname{CCSD}(\mathrm{T}) / \mathrm{CBS}$ limit with just the VDZ-F12 basis set.

In general, our calculated CCSD(T)/CBS limit oscillator strengths are in reasonably good agreement with experiment, particularly given that the oscillator strengths of the fundamental to fourth overtone transitions span several orders of magnitude. For $\mathrm{OH}$, our calculated oscillator strengths of the fundamental and first overtone transitions are slightly overestimated whereas the higher overtones are slightly underes- timated. For HF, our calculated fundamental, first and second overtone oscillator strengths are all slightly overestimated with respect to experiment. For $\mathrm{HCl}$, all of our calculated oscillator strengths are larger than experiment with the fundamental and first overtone transition overestimated by $40 \%-50 \%$ and the higher overtones overestimated by a factor of 2.

\section{B. Polyatomic frequencies}

In Tables VII-XI we present experimental and calculated $\mathrm{XH}$-stretching frequencies for $\mathrm{CH}_{3} \mathrm{OH}, \mathrm{HNO}_{3}, \mathrm{HCOOH}$, and $\mathrm{H}_{2} \mathrm{O}-\mathrm{H}_{2} \mathrm{O}$ obtained with conventional and explicitly correlated $\operatorname{CCSD}(\mathrm{T})$ potential energy curves. It is important to note that our simple 1D local mode vibrational model assumes that the $\mathrm{XH}$-stretching mode is isolated from all other vibrational modes. The appropriateness of this assumption complicates comparison of our calculated $\mathrm{XH}$-stretching transitions for these polyatomic systems to experiment. Nonetheless, the four molecular systems (and five XHstretching modes) chosen have been previously found to be at least reasonably described both theoretically and experimentally using a simple $1 \mathrm{D}$ vibrational model. ${ }^{12,46-49} \mathrm{We}$ investigate this issue further by comparing our $1 \mathrm{D} \mathrm{XH}-$ stretching frequencies to results obtained using a full $3 \mathrm{~N}-6$ (where $\mathrm{N}$ is the number of atoms) VPT2 anharmonic vibra-

TABLE VI. Conventional CCSD(T) and explicitly correlated CCSD(T)-F12b calculated and experimental oscillator strengths for hydrogen chloride.

\begin{tabular}{lccccc}
\hline \hline Basis set & $\Delta v=1$ & $\Delta v=2$ & $\Delta v=3$ & $\Delta v=4$ & $\Delta v=5$ \\
\hline AVDZ & $5.3 \times 10^{-6}$ & $1.5 \times 10^{-7}$ & $1.6 \times 10^{-9}$ & $2.1 \times 10^{-11}$ & $3.4 \times 10^{-13}$ \\
AVTZ & $6.4 \times 10^{-6}$ & $1.7 \times 10^{-7}$ & $1.5 \times 10^{-9}$ & $1.9 \times 10^{-12}$ & $2.2 \times 10^{-12}$ \\
AVQZ & $7.1 \times 10^{-6}$ & $1.7 \times 10^{-7}$ & $1.6 \times 10^{-9}$ & $6.1 \times 10^{-12}$ & $6.0 \times 10^{-13}$ \\
AV5Z & $7.3 \times 10^{-6}$ & $1.7 \times 10^{-7}$ & $1.5 \times 10^{-9}$ & $5.9 \times 10^{-12}$ & $4.7 \times 10^{-13}$ \\
AV6Z & $7.4 \times 10^{-6}$ & $1.7 \times 10^{-7}$ & $1.6 \times 10^{-9}$ & $6.3 \times 10^{-12}$ & $5.0 \times 10^{-13}$ \\
CBS $^{\text {a }}$ & $7.6 \times 10^{-6}$ & $1.8 \times 10^{-7}$ & $1.6 \times 10^{-9}$ & $6.7 \times 10^{-12}$ & $5.0 \times 10^{-13}$ \\
VDZ-F12 $^{\text {VTZ-F12 }}$ & $7.2 \times 10^{-6}$ & $1.9 \times 10^{-7}$ & $1.8 \times 10^{-9}$ & $8.7 \times 10^{-12}$ & $2.4 \times 10^{-13}$ \\
VQZ-F12 $_{\text {Expt. }}$ & $7.5 \times 10^{-6}$ & $1.8 \times 10^{-7}$ & $1.7 \times 10^{-9}$ & $9.4 \times 10^{-12}$ & $3.1 \times 10^{-13}$ \\
\hline \hline
\end{tabular}

${ }^{\mathrm{a}}$ Extrapolation to the CBS limit utilizes AV5Z and AV6Z results.

${ }^{\mathrm{b}}$ Experimental oscillator strengths from HITRAN (Ref. 62). 
TABLE VII. Conventional CCSD(T) and explicitly correlated CCSD(T)-F12b calculated and experimental $\mathrm{OH}$-stretching frequencies $\left(\right.$ in $\mathrm{cm}^{-1}$ ) for methanol.

\begin{tabular}{llllll}
\hline \hline Basis set & $\Delta v=1$ & $\Delta v=2$ & $\Delta v=3$ & $\Delta v=4$ & $\Delta v=5$ \\
\hline AVDZ & 3642 & 7108 & 10401 & 13523 & 16474 \\
AVTZ & 3676 & 7184 & 10524 & 13697 & 16701 \\
AVQZ & 3693 & 7217 & 10572 & 13759 & 16779 \\
VDZ-F12 & 3694 & 7218 & 10574 & 13761 & 16780 \\
VTZ-F12 & 3695 & 7221 & 10578 & 13768 & 16790 \\
VQZ-F12 & 3697 & 7225 & 10585 & 13777 & 16803 \\
Expt. $^{\text {a }}$ & 3683.91 & 7196.09 & 10525.6 & 13704.6 & 16705.9 \\
\hline \hline
\end{tabular}

${ }^{\mathrm{a}}$ Experimental frequencies are from Ref. 63.

tional model. The VPT2 vibrational model assumes each vibrational mode can be described using a fourth order polynomial, which when treated by second order perturbation theory gives the same energy expression as the Morse oscillator potential. ${ }^{50}$ To facilitate comparison with the VPT2 frequencies, we have also calculated the 1D local mode XHstretching frequencies using a Morse oscillator approach. ${ }^{14,51}$ We include these 1D Morse oscillator XH-stretching frequencies, the equivalent VPT2 frequencies and the difference between the 1D Morse and VPT2 results (as an estimate of the magnitude of vibrational coupling) in the supplementary deposit. ${ }^{32}$ Finally, it should be noted that the primary purpose of this paper is not to calculate $\mathrm{XH}$-stretching transitions to spectroscopic accuracy but to consider the basis set convergence of calculated frequencies and intensities of $\mathrm{XH}-$ stretching vibrational modes using conventional and explicitly correlated $\operatorname{CCSD}(\mathrm{T})$ theory. Given the excellent agreement observed for the diatomic systems in the previous section, we consider the present polyatomic $\operatorname{CCSD}(\mathrm{T})-\mathrm{F} 12 \mathrm{~b} /$ VQZ-F12 frequencies and oscillator strengths as representative of the $\operatorname{CCSD}(\mathrm{T}) / \mathrm{CBS}$ limit.

The $\mathrm{CH}_{3} \mathrm{OH}$ fundamental and overtone $\mathrm{OH}$-stretching frequencies obtained with conventional $\operatorname{CCSD}(\mathrm{T})$ converge monotonically upwards from AVDZ to AVQZ. The explicitly correlated $\mathrm{OH}$-stretching frequencies also systematically increase as the cardinal number of the basis set increases, although the variation in frequencies is much smaller than the conventional $\operatorname{CCSD}(\mathrm{T})$ results. The conventional $\operatorname{CCSD}(\mathrm{T})$ frequencies appear to converge toward the explicitly correlated results and we find that the conventional $\operatorname{CCSD}(\mathrm{T}) /$ AVQZ frequencies are in good agreement with the CCSD(T)-F12b/VDZ-F12 frequencies. The OH-stretching frequencies obtained with conventional $\operatorname{CCSD}(\mathrm{T})$ and the
AVDZ and AVTZ basis are lower than the experimentally determined values whereas the CCSD(T)/AVQZ and explicitly correlated $\operatorname{CCSD}(\mathrm{T})-\mathrm{F} 12 \mathrm{~b}$ frequencies are all higher than experiment. Furthermore, we find that the conventional $\mathrm{CCSD}(\mathrm{T}) / \mathrm{AVTZ}$ calculated $\mathrm{OH}$-stretching frequencies are in closest agreement with experiment. The $\operatorname{CCSD}(\mathrm{T})-\mathrm{F} 12 \mathrm{~b} /$ VQZ-F12 frequencies [as an estimate of the CCSD(T)/CBS limit] are overestimated by $13 \mathrm{~cm}^{-1}$ in the fundamental region and $\sim 100 \mathrm{~cm}^{-1}$ in fourth overtone region. This discrepancy with experiment is larger than but still comparable to the diatomic results in the previous section and indicates that the $\mathrm{OH}$-stretching mode of $\mathrm{CH}_{3} \mathrm{OH}$ is reasonably well localized. We find that the effect of vibrational coupling within the VPT2 model is modest and lowers the $\mathrm{OH}$-stretching frequency of $\mathrm{CH}_{3} \mathrm{OH}$ by $11 \mathrm{~cm}^{-1}$ in the fundamental region and $\sim 70 \mathrm{~cm}^{-1}$ in the fourth overtone region. Addition of this VPT2 vibrational coupling "correction" to the $\operatorname{CCSD}(\mathrm{T})$ F12b/VQZ-F12 1D OH-stretching frequencies gives results for $\mathrm{CH}_{3} \mathrm{OH}$ that are of comparable accuracy (with respect to experiment) to the diatomic frequencies in the previous section.

The fundamental and overtone $\mathrm{OH}$-stretching frequencies of $\mathrm{HNO}_{3}$ calculated with conventional $\mathrm{CCSD}(\mathrm{T})$ increase monotonically as the cardinal number of the basis set increases from AVDZ-AVQZ. In contrast, the explicitly correlated $\operatorname{CCSD}(\mathrm{T})$-F12b OH-stretching frequencies obtained with the VDZ-F12, VTZ-F12 and VQZ-F12 basis sets oscillate to a small extent. Fundamental and overtone $\mathrm{OH}-$ stretching frequencies obtained with conventional $\operatorname{CCSD}(\mathrm{T})$ appear to converge toward the explicitly correlated frequencies as the basis set increases. With exception of the conventional $\operatorname{CCSD}(\mathrm{T}) / \mathrm{AVDZ}$ results, all of our other calculated $\mathrm{OH}$-stretching frequencies are overestimated when compared

TABLE VIII. Conventional CCSD(T) and explicitly correlated CCSD(T)-F12b calculated and experimental $\mathrm{OH}$-stretching frequencies (in $\mathrm{cm}^{-1}$ ) for nitric acid.

\begin{tabular}{lccccc}
\hline \hline Basis set & $\Delta v=1$ & $\Delta v=2$ & $\Delta v=3$ & $\Delta v=4$ & $\Delta v=5$ \\
\hline AVDZ & 3534 & 6905 & 10118 & 13178 & 16088 \\
AVTZ & 3567 & 6980 & 10239 & 13348 & 16310 \\
AVQZ & 3583 & 7008 & 10280 & 13402 & 16376 \\
VDZ-F12 & 3589 & 7021 & 10300 & 13429 & 16410 \\
VTZ-F12 & 3584 & 7011 & 10285 & 13409 & 16385 \\
VQZ-F12 & 3585 & 7014 & 10289 & 13415 & 16394 \\
Expt. $^{a}$ & 3551 & $\sim 6940$ & 10173 & 13245 & 16160 \\
\hline \hline
\end{tabular}

${ }^{\mathrm{a}}$ Experimental frequencies are from Refs. 64-66. 
TABLE IX. Conventional CCSD(T) and explicitly correlated $\operatorname{CCSD}(\mathrm{T})-\mathrm{F} 12 \mathrm{~b}$ calculated and experimental $\mathrm{OH}$-stretching frequencies (in $\mathrm{cm}^{-1}$ ) for formic acid.

\begin{tabular}{llllll}
\hline \hline Basis set & $\Delta v=1$ & $\Delta v=2$ & $\Delta v=3$ & $\Delta v=4$ & $\Delta v=5$ \\
\hline AVDZ & 3557 & 6948 & 10179 & 13253 & 16174 \\
AVTZ & 3587 & 7016 & 10289 & 13409 & 16378 \\
AVQZ & 3602 & 7043 & 10328 & 13460 & 16442 \\
VDZ-F12 & 3606 & 7052 & 10342 & 13479 & 16466 \\
VTZ-F12 & 3603 & 7046 & 10332 & 13466 & 16449 \\
VQZ-F12 & 3604 & 7049 & 10337 & 13473 & 16459 \\
Expt. $^{\text {a }}$ & 3568.9 & 6968.26 & 10200 & 13284.1 & $\cdots$ \\
\hline \hline
\end{tabular}

${ }^{\mathrm{a}}$ Experimental frequencies are from Refs. 49, 52, and 57.

to the experimental values. Fortuitously, $\mathrm{OH}$-stretching frequencies for $\mathrm{HNO}_{3}$ obtained with conventional $\operatorname{CCSD}(\mathrm{T}) /$ AVDZ are in best agreement with experiment. The CCSD(T)-F12b/VQZ-F12 frequencies are overestimated by $34 \mathrm{~cm}^{-1}$ in the fundamental region and $\sim 230 \mathrm{~cm}^{-1}$ in fourth overtone region when compared to experiment. This discrepancy is much larger than the inherent overestimation error of the $\operatorname{CCSD}(\mathrm{T})$ method that was observed for the diatomic systems and suggests that the $\mathrm{OH}$-stretching mode in $\mathrm{HNO}_{3}$ is significantly coupled to the other vibrational modes. ${ }^{41} \mathrm{We}$ find that the effect of vibrational coupling within the VPT2 model is significant, lowering the $\mathrm{OH}$-stretching frequency of $\mathrm{HNO}_{3}$ by $28 \mathrm{~cm}^{-1}$ in the fundamental region and $\sim 160 \mathrm{~cm}^{-1}$ in the fourth overtone region. Thus inclusion of coupling to the other vibrational modes brings the calculated $\mathrm{OH}$-stretching frequencies for $\mathrm{HNO}_{3}$ in closer agreement with experiment.

The fundamental and overtone $\mathrm{OH}$-stretching frequencies of $\mathrm{HCOOH}$ calculated with conventional $\mathrm{CCSD}(\mathrm{T})$ are found to monotonically converge upwards as the cardinal number of the basis set increases AVDZ-AVQZ. The explicitly correlated $\operatorname{CCSD}(\mathrm{T})-\mathrm{F} 12 \mathrm{~b}$ OH-stretching frequencies do not exhibit any systematic convergence as the basis set increases, however, the oscillation from VDZ-F12 to VQZ-F12 is small. Similar to $\mathrm{HNO}_{3}$, the conventional CCSD(T) fundamental and overtone frequencies of $\mathrm{HCOOH}$ appear to converge toward the explicitly correlated results as the basis set increases. With exception of the conventional $\operatorname{CCSD}(\mathrm{T}) /$ AVDZ frequencies, all other calculated frequencies are higher than the experimentally measured values. We find that the $\operatorname{CCSD}(\mathrm{T})-\mathrm{F} 12 \mathrm{~b} / \mathrm{VQZ}-\mathrm{F} 12 \mathrm{OH}-$ stretching frequencies of $\mathrm{HCOOH}$ are overestimated by $35 \mathrm{~cm}^{-1}$ in the fundamental region and $\sim 190 \mathrm{~cm}^{-1}$ in third overtone region when com- pared to experiment. This discrepancy is much larger than the inherent overestimation error of the $\operatorname{CCSD}(\mathrm{T})$ method and suggests that like $\mathrm{HNO}_{3}$, the $\mathrm{OH}$-stretching mode in $\mathrm{HCOOH}$ is significantly coupled to the other vibrational modes. ${ }^{52}$ We find that the effect of vibrational coupling within the VPT2 model is significant, lowering the $\mathrm{OH}$ stretching frequency of $\mathrm{HCOOH}$ by $30 \mathrm{~cm}^{-1}$ in the fundamental region and $\sim 210 \mathrm{~cm}^{-1}$ in the fourth overtone region. Addition of this VPT2 anharmonic coupling correction to the calculated 1D results improves the agreement with the experimental frequencies.

The fundamental $\mathrm{CH}$-stretching frequency of $\mathrm{HCOOH}$ calculated with conventional $\operatorname{CCSD}(\mathrm{T})$ oscillates as the cardinal number of the basis set increases AVDZ-AVQZ whereas the $\mathrm{CH}$-stretching overtone frequencies all monotonically converge upwards. It is worth noting that variation in both the fundamental and overtone $\mathrm{CH}$-stretching frequencies of $\mathrm{HCOOH}$ obtained with conventional $\operatorname{CCSD}(\mathrm{T})$ is much smaller than the corresponding $\mathrm{OH}$-stretching frequencies in $\mathrm{CH}_{3} \mathrm{OH}, \mathrm{HNO}_{3}$, and $\mathrm{HCOOH}$. We find that the explicitly correlated $\mathrm{CH}$-stretching frequencies of $\mathrm{HCOOH}$ do not exhibit any systematic convergence with increasing basis set, although again the variation is small. The conventional CCSD(T)/AVQZ fundamental and overtone frequencies are found to be in good agreement with all of the explicitly correlated results. The conventional and explicitly correlated $\operatorname{CCSD}(\mathrm{T}) \mathrm{CH}$-stretching frequencies of $\mathrm{HCOOH}$ are all significantly overestimated with respect to experiment. We find that the CCSD(T)-F12b/VQZ-F12 CH-stretching frequencies of $\mathrm{HCOOH}$ are overestimated by $38 \mathrm{~cm}^{-1}$ in the fundamental region and $\sim 210 \mathrm{~cm}^{-1}$ in third overtone region when compared to experiment. Again, this relatively large discrepancy is attributed to vibrational coupling, which within the

TABLE X. Conventional CCSD(T) and explicitly correlated CCSD(T)-F12b calculated and experimental CHstretching frequencies (in $\mathrm{cm}^{-1}$ ) for formic acid.

\begin{tabular}{lccccc}
\hline \hline Basis set & $\Delta v=1$ & $\Delta v=2$ & $\Delta v=3$ & $\Delta v=4$ & $\Delta v=5$ \\
\hline AVDZ & 2979 & 5836 & 8571 & 11186 & 13681 \\
AVTZ & 2977 & 5837 & 8579 & 11206 & 13717 \\
AVQZ & 2980 & 5842 & 8587 & 11216 & 13731 \\
VDZ-F12 & 2981 & 5844 & 8591 & 11223 & 13740 \\
VTZ-F12 & 2979 & 5840 & 8585 & 11215 & 13730 \\
VQZ-F12 & 2981 & 5844 & 8591 & 11223 & 13740 \\
Expt. $^{a}$ & 2943 & 5774 & 8446 & 11012 & $\cdots$ \\
\hline \hline
\end{tabular}

${ }^{\bar{a}}$ Experimental frequencies are from Refs. 49, 52, and 57. 
TABLE XI. Conventional CCSD(T) and explicitly correlated CCSD(T)-F12b calculated OH(b)-stretching frequencies $\left(\right.$ in $\mathrm{cm}^{-1}$ ) for water dimer.

\begin{tabular}{lccccc}
\hline \hline Basis set & $\Delta v=1$ & $\Delta v=2$ & $\Delta v=3$ & $\Delta v=4$ & $\Delta v=5$ \\
\hline AVDZ $^{\text {a }}$ & 3546 & 6902 & 10069 & 13045 & 15825 \\
AVTZ $^{\text {a }}$ & 3572 & 6961 & 10162 & 13169 & 15974 \\
AVQZ $^{\text {a }}$ & 3585 & 6983 & 10193 & 13208 & 16022 \\
$\mathrm{~A}^{\prime}$ VDZ & 3564 & 6940 & 10129 & 13128 & 15933 \\
$\mathrm{~A}^{\prime}$ VTZ & 3579 & 6977 & 10190 & 13211 & 16034 \\
$\mathrm{~A}^{\prime}$ VQZ & 3588 & 6991 & 10204 & 13224 & 16043 \\
$\mathrm{~A}^{\prime}$ V5Z & 3591 & 6996 & 10212 & 13235 & 16056 \\
VDZ-F12 $_{\text {VTZ-F12 }}^{3597}$ & 7010 & 10235 & 13269 & 16101 \\
VQZ-F12 & 3589 & 6994 & 10210 & 13233 & 16055 \\
Expt. & 3591 & 6996 & 10214 & 13238 & 16061 \\
\hline \hline
\end{tabular}

${ }^{\mathrm{a}}$ From Ref. 46.

${ }^{\mathrm{b}}$ Experimental frequencies are from Refs. 55 and 56.

VPT2 model is found to lower the $\mathrm{CH}$-stretching frequency of $\mathrm{HCOOH}$ by $60 \mathrm{~cm}^{-1}$ in the fundamental region and $\sim 340 \mathrm{~cm}^{-1}$ in the fourth overtone region.

The final polyatomic system considered is the $\mathrm{H}_{2} \mathrm{O}-\mathrm{H}_{2} \mathrm{O}$ complex, where we consider only the vibrational frequencies of the hydrogen bonded $\mathrm{OH}(\mathrm{b})$-stretching mode. The accurate calculation of vibrational frequencies in molecular complexes is further complicated by basis set superposition error, which artificially increases the interaction energy and hence overestimates the effects of complexation. ${ }^{53,54}$ Recently, we showed that basis set superposition error could be significantly reduced by including diffuse basis functions only on the heavy atoms (i.e., all atoms but hydrogen) in a series of hydrogen bonded complexes, including $\mathrm{H}_{2} \mathrm{O}-\mathrm{H}_{2} \mathrm{O}{ }^{23}$ The fundamental and overtone $\mathrm{OH}(\mathrm{b})$-stretching frequencies of $\mathrm{H}_{2} \mathrm{O}-\mathrm{H}_{2} \mathrm{O}$ obtained with conventional $\operatorname{CCSD}(\mathrm{T})$ and both the fully (AVDZAVQZ) and partially (A'VDZ-A'V5Z) augmented basis sets converge upwards as the cardinal number of the basis set is increased. Consistent with the previous monomeric systems investigated, the $\mathrm{OH}(\mathrm{b})$-stretching frequencies obtained with the explicitly correlated $\operatorname{CCSD}(\mathrm{T})-\mathrm{F} 12 \mathrm{~b}$ method oscillate to a small extent from VDZ-F12 to VQZ-F12. For a given cardinal number, we find that fundamental and overtone $\mathrm{OH}(\mathrm{b})$ stretching frequencies obtained with the partially augmented basis sets are in better agreement with the explicitly correlated results than frequencies obtained with the fully augmented basis sets. This indicates, as expected, that the effect of basis set superposition error on the $\mathrm{OH}(\mathrm{b})$-stretching frequencies is reduced by restricting diffuse basis functions to the heavy atoms.

There are limited experimental results available for the $\mathrm{OH}(\mathrm{b})$-stretching frequencies of $\mathrm{H}_{2} \mathrm{O}-\mathrm{H}_{2} \mathrm{O}$, with only the fundamental transition observed in gas phase and the first overtone transition observed in a $\mathrm{Ne}$ matrix. ${ }^{55,56}$ In contrast to the other polyatomic systems, all of our calculated frequencies for the $\mathrm{H}_{2} \mathrm{O}-\mathrm{H}_{2} \mathrm{O}$ complex are underestimated with respect to the experimental values. We find that the CCSD(T)-F12b/VQZ-F12 OH(b)-stretching frequencies of $\mathrm{H}_{2} \mathrm{O}-\mathrm{H}_{2} \mathrm{O}$ are underestimated by $10 \mathrm{~cm}^{-1}$ in the fundamental region and $22 \mathrm{~cm}^{-1}$ in the first overtone region when compared to experiment. We have previously shown that the fundamental $\mathrm{OH}(\mathrm{b})$-stretching frequency of $\mathrm{H}_{2} \mathrm{O}-\mathrm{H}_{2} \mathrm{O}$ calculated using a 1D vibrational model is lowered by $9 \mathrm{~cm}^{-1}$ by inclusion of intramolecular vibrational coupling calculated with a harmonically coupled anharmonic oscillator (HCAO) vibrational model. ${ }^{46}$ However, vibrational coupling to the remaining intermolecular vibrational modes is also important and the VPT2 fundamental $\mathrm{OH}(\mathrm{b})$-stretching frequency is found to be $17 \mathrm{~cm}^{-1}$ higher in energy than the 1D approach. ${ }^{42,46}$ It follows that for $\mathrm{H}_{2} \mathrm{O}-\mathrm{H}_{2} \mathrm{O}$, unlike the other polyatomic systems, the net effect of vibrational coupling is to increase the $1 \mathrm{D} \mathrm{OH}(\mathrm{b})$-stretching frequency.

In summary, we find that the basis set dependence of calculated frequencies obtained with the explicitly correlated $\operatorname{CCSD}(\mathrm{T})-\mathrm{F} 12 \mathrm{~b}$ method is much smaller than with conventional $\operatorname{CCSD}(\mathrm{T})$ and even $\operatorname{CCSD}(\mathrm{T})-\mathrm{F} 12 \mathrm{~b} / \mathrm{VDZ}-\mathrm{F} 12$ frequencies are in good agreement with results obtained with much larger basis sets. The computational demands of the CCSD(T)-F12b/VDZ-F12 method are not excessive and as such its use with full $3 \mathrm{~N}-6$ multidimensional anharmonic vibrational models such as VSCF, VCI, VCC, and VPT2 should be practicable for systems up to ten atoms. However, it is important to remember that the accuracy of any $\operatorname{CCSD}(\mathrm{T})-\mathrm{F} 12 \mathrm{~b}$ multidimensional anharmonic frequencies will still be limited to the inherent accuracy of the $\operatorname{CCSD}(\mathrm{T})$ method, which we found to be $4-6 \mathrm{~cm}^{-1}$ for fundamental frequencies and $26-46 \mathrm{~cm}^{-1}$ for fourth overtone transitions in the previous section. When multidimensional anharmonic models are not practicable and instead a local mode vibrational model is used we suggest calculating the corresponding potential energy surfaces at the CCSD(T)/AVTZ level of theory. With conventional $\operatorname{CCSD}(\mathrm{T})$, meaningful results can only be expected with a triple- $\zeta$ or larger basis set ${ }^{38}$ and the additional computational demands of a quadruple- $\zeta$ basis set are not warranted when using a vibrational model that neglects coupling to the other modes. We find that even for the well-localized vibrational modes considered here, the inclusion of vibrational coupling has a much greater effect on the calculated frequencies than increasing the basis set from AVTZ to AVQZ. 
TABLE XII. Explicitly correlated CCSD(T)-F12b/VQZ-F12 calculated and experimental XH-stretching oscillator strengths $\left(\right.$ in $\mathrm{cm}^{-1}$ ).

\begin{tabular}{|c|c|c|c|c|c|}
\hline & $\Delta v=1$ & $\Delta v=2$ & $\Delta v=3$ & $\Delta v=4$ & $\Delta v=5$ \\
\hline \multicolumn{6}{|c|}{$\mathrm{CH}_{3} \mathrm{OH}^{\mathrm{a}}$} \\
\hline Calc. & $3.6 \times 10^{-6}$ & $6.2 \times 10^{-7}$ & $2.9 \times 10^{-8}$ & $1.9 \times 10^{-10}$ & $2.0 \times 10^{-10}$ \\
\hline Expt. & $(2.9-3.7) \times 10^{-6}$ & $(3.1-6.3) \times 10^{-7}$ & $(2.7-4.7) \times 10^{-8}$ & $(1.9-2.5) \times 10^{-10}$ & $4.0 \times 10^{-10}$ \\
\hline \multicolumn{6}{|c|}{$\mathrm{HNO}_{3}{ }^{\mathrm{b}}$} \\
\hline Calc. & $1.6 \times 10^{-5}$ & $6.3 \times 10^{-7}$ & $2.8 \times 10^{-8}$ & $2.4 \times 10^{-9}$ & $2.9 \times 10^{-10}$ \\
\hline Expt. & $(1.1-1.6) \times 10^{-5}$ & $(3.8-7.8) \times 10^{-7}$ & $(3.0-3.3) \times 10^{-8}$ & $(2.5-3.2) \times 10^{-9}$ & $2.9 \times 10^{-10}$ \\
\hline \multicolumn{6}{|c|}{$\mathrm{HCOOH} \mathrm{OH}$-stretching ${ }^{c}$} \\
\hline Calc. & $1.0 \times 10^{-5}$ & & & & \\
\hline Expt. & $1.6 \times 10^{-5}$ & & & & \\
\hline \multicolumn{6}{|c|}{ HCOOH CH-stretching ${ }^{\mathrm{c}}$} \\
\hline Calc. & $2.6 \times 10^{-6}$ & & & & \\
\hline Expt. & $9.6 \times 10^{-6}$ & & & & \\
\hline \multicolumn{6}{|c|}{$\mathrm{H}_{2} \mathrm{O}-\mathrm{H}_{2} \mathrm{O}^{\mathrm{d}}$} \\
\hline Calc. & $6.9 \times 10^{-5}$ & $2.0 \times 10^{-8}$ & $2.6 \times 10^{-9}$ & $6.6 \times 10^{-10}$ & $1.1 \times 10^{-10}$ \\
\hline Expt. & $2.5 \times 10^{-5}$ & & & & \\
\hline
\end{tabular}

\section{Polyatomic intensities}

In Table XII, we present CCSD(T)-F12b/VQZ-F12 calculated and experimental fundamental and overtone $\mathrm{XH}-$ stretching oscillator strengths for $\mathrm{CH}_{3} \mathrm{OH}, \mathrm{HNO}_{3}, \mathrm{HCOOH}$, and $\mathrm{H}_{2} \mathrm{O}-\mathrm{H}_{2} \mathrm{O}$. The calculated AVDZ, AVTZ, and AVQZ conventional $\operatorname{CCSD}(\mathrm{T})$ oscillator strengths and the calculated VDZ-F12 and VTZ-F12 explicitly correlated CCSD(T)-F12 oscillator strengths are included in the supplementary deposit. ${ }^{32}$ The measurement of absolute oscillator strengths is inherently more difficult than the measurement of absolute absorption frequencies, particularly for overtone transitions. As a result, different experimental investigations and indeed different experimental techniques often result in appreciably different absolute oscillator strengths. To reflect this uncertainty, where possible, we have shown a range of different experimental oscillator strengths in Table XII.

In general, we find that our CCSD(T)-F12b/VQZ-F12 calculated $\mathrm{XH}$-stretching oscillator strengths are in very good agreement with experiment. For $\mathrm{CH}_{3} \mathrm{OH}$, we find that the calculated fundamental, first, second and third overtone $\mathrm{OH}$-stretching oscillator strengths are within the range of experimentally determined oscillator strengths. The fourth overtone $\mathrm{OH}$-stretching oscillator strength of $\mathrm{CH}_{3} \mathrm{OH}$ is a factor of 2 smaller than experiment. For $\mathrm{HNO}_{3}$, we find that the calculated fundamental, first, second and fourth overtone $\mathrm{OH}$-stretching oscillator strengths are within the range of experimentally determined oscillator strengths. Our calculated third overtone $\mathrm{OH}$-stretching oscillator strength of $\mathrm{HNO}_{3}$ is approximately $4 \%$ smaller than the lowest experimental value.

While there have been several experimental investigations of the fundamental and overtone $\mathrm{CH}-$ and $\mathrm{OH}-$ stretching frequencies of $\mathrm{HCOOH},{ }^{49,52,57}$ only the fundamen- tal oscillator strengths have been determined. ${ }^{58}$ Formic acid has a strong tendency to form dimers and higher order clusters in the gas phase, which makes it very difficult to independently measure the concentration of $\mathrm{HCOOH}$ monomer and hence determine the oscillator strength of the monomeric absorption bands. We find that our calculated fundamental $\mathrm{OH}$-stretching oscillator strength of $\mathrm{HCOOH}$ is $\sim 40 \%$ smaller than the experimentally determined oscillator strength. For the $\mathrm{CH}$-stretching mode of $\mathrm{HCOOH}$, we find that our calculated fundamental oscillator strength is nearly a factor of 4 smaller than experiment. It is possible that the effect of dimerization has been overestimated in the experimental $\mathrm{HCOOH}$ investigation, and that the true concentration of monomeric $\mathrm{HCOOH}$ was actually higher, resulting in lower experimental oscillator strengths. However, because the calculated $\mathrm{OH}$-stretching oscillator strength is underestimated by $\sim 40 \%$ and the calculated $\mathrm{CH}$-stretching oscillator strength underestimated by a factor of 4 , a change in the monomeric $\mathrm{HCOOH}$ concentration would only bring one of these two calculated oscillator strengths into agreement with experiment. It is worth noting that this discrepancy between theory and experiment is unlikely due to our simple $1 \mathrm{D}$ vibrational model, with VPT2 $\mathrm{CH}$ - and $\mathrm{OH}$-stretching fundamental oscillator strengths in excellent agreement (differing by less than $2 \%$ ) with $1 \mathrm{D}$ results at the CCSD(T)/AVTZ level of theory. The OH-stretching frequency of $\mathrm{HCOOH}$ is significantly redshifted upon dimerization whereas the $\mathrm{CH}$ stretching frequency is only mildly blueshifted such that the $\mathrm{CH}$-stretching rotational profile of $\mathrm{HCOOH}$ and $(\mathrm{HCOOH})_{2}$ overlap significantly. ${ }^{59,60}$ Hence, the intensity of the weak $\mathrm{CH}$-stretching transition is likely to be less accurately determined than the corresponding $\mathrm{OH}$-stretching transition.

The fundamental $\mathrm{OH}(\mathrm{b})$-stretching oscillator strength of 
$\mathrm{H}_{2} \mathrm{O}-\mathrm{H}_{2} \mathrm{O}$ measured using $\mathrm{He}$ droplet isolation techniques is nearly a factor of 3 smaller than our calculated 1D oscillator strength. ${ }^{61}$ We have previously shown at the $\operatorname{CCSD}(\mathrm{T}) / \mathrm{AVTZ}$ level of theory that the fundamental $\mathrm{OH}(\mathrm{b})$-stretching oscillator strength of $\mathrm{H}_{2} \mathrm{O}-\mathrm{H}_{2} \mathrm{O}$ calculated using a 1D vibrational model is lowered by $20 \%\left(5.7 \times 10^{-5}\right)$ by inclusion of intramolecular vibrational coupling calculated using a HCAO vibrational model..$^{46}$ The effect of vibrational coupling to the remaining intermolecular vibrational modes has been shown to be even more important with the $\operatorname{CCSD}(\mathrm{T}) / \mathrm{AVTZ}$ fundamental VPT2 oscillator strength calculated to be $2.8 \times 10^{-5}$, in good agreement with experiment. ${ }^{42}$

\section{CONCLUSIONS}

We have calculated $\mathrm{XH}$-stretching fundamental and overtone transitions of $\mathrm{OH}, \mathrm{HF}, \mathrm{HCl}, \mathrm{CH}_{3} \mathrm{OH}, \mathrm{HNO}_{3}$, $\mathrm{HCOOH}$, and $\mathrm{H}_{2} \mathrm{O}-\mathrm{H}_{2} \mathrm{O}$ with a 1D local mode vibrational model. The necessary $\mathrm{XH}$-stretching potential energy and dipole moment curves were calculated $a b$ initio using conventional $\operatorname{CCSD}(\mathrm{T})$ theory and the newly developed explicitly correlated CCSD(T)-F12 method. We find that the basis set convergence of calculated frequencies and oscillator strengths obtained with the explicitly correlated method is much more rapid than conventional $\operatorname{CCSD}(\mathrm{T})$. In general, CCSD(T)-F12 results obtained with a given basis set were found to be of comparable accuracy to conventional $\operatorname{CCSD}(\mathrm{T})$ results obtained with a basis set two cardinal numbers larger. For the three diatomic systems, $\operatorname{CCSD}(\mathrm{T})$-F12 frequencies and oscillator strengths obtained with the VTZF12 and VQZ-F12 basis sets were found to be in excellent agreement with the CCSD(T)/CBS limit with the VDZ-F12 results only slightly less accurate.

The impressive basis set convergence of the explicitly correlated CCSD(T)-F12 method unfortunately exposes the inherent error of the $\operatorname{CCSD}(\mathrm{T})$ method to overestimate frequencies with respect to experiment, which is normally compensated by basis set incompleteness error. To obtain XHstretching frequencies in closest agreement with experiment we suggest use of conventional $\operatorname{CCSD}(\mathrm{T})$ with the aug-ccpVTZ or aug-cc-pVQZ basis set. This combination of method and basis set appears to fortuitously balance the inherent overestimation error of the $\operatorname{CSCD}(\mathrm{T})$ method with the inherent underestimation error due to basis set incompleteness.

The calculated $\operatorname{CCSD}(\mathrm{T})$-F12 XH-stretching frequencies of the diatomic systems were found to be in much better agreement with experiment than the corresponding $\mathrm{XH}$ stretching frequencies of the polyatomic systems. This indicates that even for reasonably well-localized oscillators, inclusion of coupling to other vibrational modes is necessary to accurately calculate vibrational frequencies. Nonetheless we find that in general, calculated 1D local mode XH-stretching oscillator strengths obtained with the CCSD(T)-F12 method are in very good agreement with experiment.

\section{ACKNOWLEDGMENTS}

We are grateful to Jeppe Olsen for use of his ONEDIM program, A. Helena Södegren for helpful discussions, and
John Stanton for assistance with running the VPT2 calculations in CFOUR. We acknowledge the Marsden Fund administered by the Royal Society of New Zealand for financial support.

${ }^{1}$ T. Helgaker, T. A. Ruden, P. Jørgensen, J. Olsen, and W. Klopper, J. Phys. Org. Chem. 17, 913 (2004).

${ }^{2}$ A. Halkier, H. Koch, P. Jørgensen, O. Christiansen, I. M. B. Nielsen, and T. Helgaker, Theor. Chem. Acc. 97, 150 (1997).

${ }^{3}$ T. Helgaker, J. Gauss, P. Jørgensen, and J. Olsen, J. Chem. Phys. 106, 6430 (1997).

${ }^{4}$ W. Kutzelnigg, Theor. Chim. Acta 68, 445 (1985).

${ }^{5}$ D. P. Tew, W. Klopper, C. Neiss, and C. Hättig, Phys. Chem. Chem. Phys. 9, 1921 (2007).

${ }^{6}$ O. Marchetti and H.-J. Werner, Phys. Chem. Chem. Phys. 10, 3400 (2008).

${ }^{7}$ G. Adler, T. B. Knizia, and H.-J. Werner, J. Chem. Phys. 127, 221106 (2007).

${ }^{8}$ G. Rauhut, G. Knizia, and H.-J. Werner, J. Chem. Phys. 130, 054105 (2009).

${ }^{9}$ B. R. Henry and H. G. Kjaergaard, Can. J. Chem. 80, 1635 (2002).

${ }^{10}$ B. R. Henry, Acc. Chem. Res. 10, 207 (1977).

${ }^{11}$ B. R. Henry, Acc. Chem. Res. 20, 429 (1987).

${ }^{12}$ H. G. Kjaergaard, J. Phys. Chem. A 106, 2979 (2002).

${ }^{13}$ H. G. Kjaergaard, J. R. Lane, A. L. Garden, D. P. Schofield, T. W. Robinson, and M. J. Mills, Adv. Quantum Chem. 55, 137 (2008).

${ }^{14}$ D. L. Howard, P. Jørgensen, and H. G. Kjaergaard, J. Am. Chem. Soc. 127, 17096 (2005).

${ }^{15}$ J. L. Fry, J. Matthews, J. R. Lane, C. M. Roehl, A. Sinha, H. G. Kjaergaard, and P. O. Wennberg, J. Phys. Chem. A 110, 7072 (2006).

${ }^{16}$ J. R. Lane, H. G. Kjaergaard, K. P. Plath, and V. Vaida, J. Phys. Chem. A 111, 5434 (2007).

${ }^{17}$ B. J. Miller, D. L. Howard, J. R. Lane, H. G. Kjaergaard, M. E. Dunn, and V. Vaida, J. Phys. Chem. A 113, 7576 (2009).

${ }^{18}$ A. B. McCoy, J. L. Fry, J. S. Francisco, A. K. Mollner, and M. Okumura, J. Chem. Phys. 122, 104311 (2005).

${ }^{19}$ J. Matthews and A. Sinha, J. Chem. Phys. 122, 104313 (2005).

${ }^{20}$ D. P. Schofield, H. G. Kjaergaard, J. Matthews, and A. Sinha, J. Chem. Phys. 123, 134318 (2005).

${ }^{21}$ T. H. Dunning, Jr., J. Chem. Phys. 90, 1007 (1989).

${ }^{22}$ A. K. Wilson and T. H. Dunning, Jr., J. Chem. Phys. 119, 11712 (2003).

${ }^{23}$ J. R. Lane and H. G. Kjaergaard, J. Chem. Phys. 131, 034307 (2009).

${ }^{24}$ H.-J. Werner, P. J. Knowles, R. Lindh et al., MOLPRO, version 2008 1, a package of ab initio programs, see http://www.molpro.net.

${ }^{25}$ K. A. Peterson, T. B. Adler, and H.-J. Werner, J. Chem. Phys. 128, 084102 (2008).

${ }^{26}$ F. R. Manby, J. Chem. Phys. 119, 4607 (2003).

${ }^{27}$ H.-J. Werner, T. B. Adler, and F. R. Manby, J. Chem. Phys. 126, 164102 (2007).

${ }^{28}$ F. Weigend, A. Köhn, and C. Hättig, J. Chem. Phys. 116, 3175 (2002).

${ }^{29}$ F. Weigend, Phys. Chem. Chem. Phys. 4, 4285 (2002).

${ }^{30}$ K. E. Yousaf and K. A. Peterson, J. Chem. Phys. 129, 184108 (2008).

${ }^{31}$ G. Knizia, T. B. Adler, and H.-J. Werner, J. Chem. Phys. 130, 054104 (2009).

${ }^{32}$ See supplementary material at http://dx.doi.org/10.1063/1.3408192 for the equivalent explicitly correlated $\operatorname{CCSD}(\mathrm{T})$-F12a XH-stretching frequencies and oscillator strengths; the conventional and explicitly correlated XH-stretching oscillator strengths of the polyatomic systems; CCSD(T)/aug-cc-pVTZ VPT2 and 1D Morse oscillator XH-stretching frequencies.

${ }^{33}$ P. W. Atkins and R. S. Friedman, Molecular Quantum Mechanics (Oxford University Press, Oxford, 1997).

${ }^{34}$ H. G. Kjaergaard, H. Yu, B. J. Schattka, B. R. Henry, and A. W. Tarr, J. Chem. Phys. 93, 6239 (1990).

${ }^{35} \mathrm{~J}$. Olsen, ONEDIM program, private communication (2005).

${ }^{36}$ H. G. Kjaergaard and B. R. Henry, J. Chem. Phys. 96, 4841 (1992).

${ }^{37}$ G. R. Low and H. G. Kjaergaard, J. Chem. Phys. 110, 9104 (1999).

${ }^{38}$ T. Helgaker, P. Jørgensen, and J. Olsen, Molecular Electronic Structure Theory (Wiley, Chichester, 2000).

${ }^{39}$ A. Halkier, T. Helgaker, P. Jørgensen, W. Klopper, H. Koch, J. Olsen, and A. K. Wilson, Chem. Phys. Lett. 286, 243 (1998).

${ }^{40}$ A. Halkier, W. Klopper, T. Helgaker, and P. Jørgensen, J. Chem. Phys. 111, 4424 (1999). 
${ }^{41}$ K. J. Feierabend, D. K. Havey, M. E. Varner, J. F. Stanton, and V. Vaida, J. Chem. Phys. 124, 124323 (2006).

${ }^{42}$ H. G. Kjaergaard, A. L. Garden, G. Chaban, R. B. Gerber, D. A. Matthews, and J. F. Stanton, J. Phys. Chem. A 112, 4324 (2008).

${ }^{43}$ J. F. Stanton, J. Gauss, M. E. Harding, and P. G. Szalay, CFour, for the current version, see http://www.cfour.de.

${ }^{44}$ F. Pawłowski, A. Halkier, P. Jørgensen, K. L. Bak, T. Helgaker, and W. Klopper, J. Chem. Phys. 118, 2539 (2003).

${ }^{45}$ T. A. Ruden, T. Helgaker, P. Jørgensen, and J. Olsen, J. Chem. Phys. 121, 5874 (2004).

${ }^{46}$ D. P. Schofield, J. R. Lane, and H. G. Kjaergaard, J. Phys. Chem. A 111 567 (2007).

${ }^{47}$ H. L. Fang, D. M. Meister, and R. L. Swofford, J. Phys. Chem. 88, 405 (1984).

${ }^{48}$ K. Takahashi, M. Sugawara, and S. Yabushita, J. Phys. Chem. A 109, 4242 (2005)

${ }^{49}$ D. Hurtmans, F. Herregodts, M. Herman, J. Lievin, A. Campargue, A Garnache, and A. A. Kachanov, J. Chem. Phys. 113, 1535 (2000).

${ }^{50}$ I. M. Mills, in Modern Spectroscopy: Modern Research, edited by K. N. Rao and C. W. Matthews (Academic, New York, 1972), pp. 115-140.

${ }^{51}$ G. Herzberg, Molecular Spectra and Molecular Structure I. Spectra of Diatomic Molecules (D. Van Nostrand, New Jersey, 1950).

${ }^{52}$ D. L. Howard and H. G. Kjaergaard, J. Chem. Phys. 121, 136 (2004).

${ }^{53}$ S. Simon, M. Duran, and J. J. Dannenberg, J. Chem. Phys. 105, 11024 (1996).

${ }^{54}$ S. Simon, J. Bertran, and M. Sodupe, J. Phys. Chem. A 105, 4359 (2001).

${ }^{55}$ F. Huisken, M. Kaloudis, and A. Kulcke, J. Chem. Phys. 104, 17 (1996).

${ }^{56}$ J. P. Perchard, Chem. Phys. 273, 217 (2001).

${ }^{57}$ M. Freytes, D. Hurtmans, S. Kassi, J. Lievin, J. Vander Auwera, A. Campargue, and M. Herman, Chem. Phys. 283, 47 (2002).

${ }^{58}$ D. Berckmans, H. P. Figeys, and P. Geerlings, J. Phys. Chem. 92, 61 (1988)
${ }^{59}$ Y. Maréchal, J. Chem. Phys. 87, 6344 (1987)

${ }^{60}$ G. M. Florio, T. S. Zwier, E. M. Myshakin, K. D. Jordan, and E. L. Sibert, J. Chem. Phys. 118, 1735 (2003).

${ }^{61}$ M. N. Slipchenko, K. E. Kuyanov, B. G. Sartakov, and A. F. Vilesov, J. Chem. Phys. 124, 241101 (2006).

${ }^{62}$ L. S. Rothman, I. E. Gordon, A. Barbe, D. C. Benner, P. F. Bernath, M. Birk, V. Boudon, L. R. Brown, A. Campargue, J. P. Champion, K. Chance, L. H. Coudert, V. Dana, V. M. Devi, S. Fally, J.-M. Flaud, R. R. Gamache, A. Goldman, D. Jacquemart, I. Kleiner, N. Lacome, W. J. Lafferty, J.-Y. Mandin, S. T. Massie, S. N. Mikhailenko, C. E. Miller, N. Moazzen-Ahmadi, O. V. Naumenko, A. V. Nikitin, J. Orphal, V. I. Perevalov, A. Perrin, A. Predoi-Cross, C. P. Rinsland, M. Rotger, M. Šimečková, M. A. H. Smith, K. Sung, S. A. Tashkun, J. Tennyson, R. A. Toth, A. C. Vandaele, and J. Vander Auwera, J. Quant. Spectrosc. Radiat. Transf. 110, 533 (2009).

${ }^{63}$ D. Rueda, O. V. Boyarkin, T. R. Rizzo, A. Chirokolava, and D. S. Perry, J. Chem. Phys. 122, 044314 (2005).

${ }^{64}$ K. J. Feierabend, D. K. Havey, and V. Vaida, Spectrochim. Acta, Part A 60, 2775 (2004).

${ }^{65}$ D. J. Donaldson, J. J. Orlando, S. Amann, G. S. Tyndall, R. J. Proos, B. R. Henry, and V. Vaida, J. Phys. Chem. A 102, 5171 (1998).

${ }^{66}$ S. S. Brown, R. W. Wilson, and A. R. Ravishankara, J. Phys. Chem. A 104, 4976 (2000).

${ }^{67}$ J. A. Phillips, J. J. Orlando, G. S. Tyndall, and V. Vaida, Chem. Phys. Lett. 296, 377 (1998).

${ }^{68}$ K. R. Lange, N. P. Wells, K. S. Plegge, and J. A. Phillips, J. Phys. Chem. A 105, 3481 (2001).

${ }^{69}$ G. J. Fleming, Postgraduate diploma in Science, University of Otago, 2001.

${ }^{70}$ R. Schofield, Bachelor of Science (Hons.), University of Otago, 1999.

${ }^{71}$ H. Zhang, C. M. Roehl, S. P. Sander, and P. O. Wennberg, J. Geophys. Res. 105, 593 (2000) 